\title{
Naïve human iPS cells
}

Center and the Tel Aviv University Sackler Faculty of Medicine.

The team generated human iPS cells with doxycycline-inducible expression of a basket of transgenes known to promote pluripotency: OCT4, SOX2, KLF4 and $c-M y c(M Y C)$.

Next, the group screened for a combination of small molecules and cytokines in culture that maintained cell pluripotency after the removal of doxycycline and, thus, doxycycline-induced expression of the transgenes.

The researchers found a handful of small molecules and cytokines By Lauren Martz, Staff Writer

The inability to generate fully undifferentiated human induced pluripotent stem cells in vitro has dogged the development of stem cell-based platforms because the residual lineage bias of the cells most likely contributes to the inefficiency and inconsistency of current differentiation protocols. An Israeli team thinks it has solved the problem with the optimization of a small molecule and cytokine cocktail capable of maintaining human cells in a more fully undifferentiated state. ${ }^{1}$

The group expects that the naïve cells will be useful in generating somatic cells for research and clinical applications and for creating new animal models, although it still remains unclear whether the cells provide an advantage over conventionally derived induced pluripotent stem (iPS) cells.

In vitro, human iPS cells have been shown to be rather heterogeneous and to exhibit varying residual lineage characteristics-even when taken from the same patient. ${ }^{2}$ Residual lineage bias of iPS cells is reflected in DNA methylation patterns, transcriptional profiles with upregulated lineage commitment genes and $\mathrm{X}$-chromosome inactivation. ${ }^{3,4}$

\section{"If this process of} maintaining naïve iPS cells is reproducible and can be applied to other species such as pigs, cows and nonhuman primates, one could imagine genome manipulation of these types of animals to develop disease models [as] very valuable for research." -Sheng Ding, University of California, San Francisco
These varying genetic and epigenetic properties of iPS cell lines most likely are the reason why existing protocols for their differentiation into somatic lineages can be inefficient and inconsistent.

In addition, and although human pluripotent stem cells, albeit of limited stability, have been generated in a primed state in vitro using transgene expression, mouse pluripotent stem cells have been shown to exhibit an earlier, more undifferentiated state in vitro. ${ }^{4}$ This raises the question of how far human pluripotent stem cell dedifferentiation can be taken.

"The question of whether a ground state actually exists in human pluripotent stem cells and not just in cells from rodents is very interesting," said Sheng Ding, a professor of pharmaceutical chemistry at the University of California, San Francisco and a senior investigator at the university's Gladstone Institute of Cardiovascular Disease.

Now, Jacob Hanna and colleagues at the Weizmann Institute of Science have developed a method for generating human iPS cells in a naïve ground state and maintaining them undifferentiated by using a combination of small molecules.

Hanna is a principal investigator at the Weizmann Institute. The paper also included researchers from the Tel Aviv Sourasky Medical medium-derived, naïve human iPS cells an naïve mouse pluripotent stem cells, which further suggested that the human cells were truly undifferentiated.

Moreover, the naïve human iPS cells had upregulated pluripotency genes and downregulated lineage commitment genes compared with primed human iPS cells. The naïve cells also had high single-cell cloning efficiency.

Finally, the group injected labeled, naïve human iPS cells into mouse morulas, which were then grown in female mice for seven to eight days. Ex vivo, the embryos had signs of chimerism, and the human cells incorporated into the different tissues during early organogenesis. These findings further suggest a highly undifferentiated cell state.

Results were published in Nature.

\section{Process benefits}

Compared with previous attempts to maintain ground-state pluripotency in human stem cells, the Weizmann team's work may help with generating transgene-independent, stable, naïve human iPS cells.

"Previous attempts to generate naïve human iPS cells relied on the use of transgenes that integrate into the genome. Not only do these transgenes encode powerful oncogenes that themselves drive cancer in 
other contexts, but the integrations themselves can be oncogenic and preclude clinical applications," said Alejandro De Los Angeles, an MD and PhD candidate at Harvard Medical School.

Matthew Vincent, director of business development at Advanced Cell Technology Inc., added that "a very elegant aspect of this approach is that rather than worrying about picking the best iPS cell induction strategy to modify, they solved the final steps, and their approach can be applied to a whole range of induction techniques to predictably stabilize the cells."

He added, "They specifically tested the approach on pluripotent stem cells formed with transgene induction techniques. It would be interesting to see whether this approach also applies to other induction techniques such as chemical, protein, microRNA and mRNA. I expect that it will."

Advanced Cell has an ESC-derived retinal pigment epithelium cell therapy in Phase I testing to treat dry age-related macular degeneration (AMD) and Stargardt macular dystrophy.

Ding said that it was good that the researchers reported stability over many passages. "The issue of stability is critical for people working on this question. Without genetic manipulation, the chemical cocktail in which you culture the cells usually decreases the cell count with each passage, and the culture is not very stable. If, indeed, these conditions maintain long-term passage, they would overcome an important hurdle," he said.

According to Vincent, "Stem cells should be able to be passaged over and over again. They should be an inexhaustible cell source, but generally this is not the case for iPS cells. They experience replicative gene senescence over time and eventually develop genetic instability."

"There are certain cell types such as blood cells and germ cells that have been very difficult to make using conventional human pluripotent stem cells. It will be worth exploring whether the described naïve human pluripotent stem cells or future, more fully naïve pluripotent stem cells will enhance access to these elusive lineages."

- Alejandro De Los Angeles, Harvard Medical School
He added, "There is still a relatively large greenfield opportunity out there for whoever develops an iPS cell technique that gives good stability without the replicative capacity issues."

Chris Parker, VP and chief commercial officer at Cellular Dynamics International Inc., said that it will be "interesting to see if they can grow large quantities of the cells while maintaining the undifferentiated state. For any applications for these cells, we will need a lot of them for practical use."

Cellular Dynamics markets the iCell platform for differentiating iPS cells into homogeneous, pure target cell types. The company also markets human iPS cell-derived cardiomyocytes, endothelial cells, neurons and hepatocytes, and it expects dopaminergic neurons to be available in 1Q14.

Hanna added that the naïve cells are also more amenable to genetic manipulation than primed iPS cells.

De Los Angeles did note that the Weizmann team's process involves complicated culture conditions.

"The culture conditions used to maintain these cells are complex, and this may impede the study of the mechanisms regulating naïve pluripotency and the widespread application of this technology," he said.
Emile Nuwaysir, COO of Cellular Dynamics, added, "These cells are at this time a little harder to culture and handle and are less stable commercial applications.

Parker told SciBX, "We need to look for replication and reproduction of the approach to see if other groups can get the same cell types. Replication is so often not possible."

\section{In vitro applications} accomplish that could not be done before. Hanna said that the new pproach could lead to iPS cells that are more homogeneous, grow faster and have single-cell cloning efficacy that makes them more amenable to genetic manipulation.

"We will be interested in seeing whether the cells that start in a more naïve state can be used to produce somatic cell lines with better properties. Are they more mature or do they have different functions?” asked Parker. "To date, this hasn't been documented."

Hanna agreed. He said that his team's next step is studying these cells and protocols for differentiation into various somatic cell lineages. These studies should show whether the new naïve cell type leads to improvements in quantity or quality of the resulting somatic cell products.

"There are certain cell types such as blood cells and germ cells that have been very difficult to make using conventional human pluripotent stem cells. It will be worth exploring whether the described naïve human pluripotent stem cells or future, more fully naïve pluripotent stem cells will enhance access to these elusive lineages," said De Los Angeles.

Ding was less sanguine that the new approach would yield improved downstream cell products. "It is very unclear if there will be benefits for in vitro pluripotent stem cell applications," he said. "Mouse embryonic cells at a naive state and at a later pluripotent state have both been used for in vitro applications with no real advantage of using one particular state over another."

Parker and Nuwaysir said that they would consider adopting the new technology if the findings are validated and if the approach is scalable and cost effective.

"We are quick to adopt any improvements that make the process of deriving cells from iPS cells better. If improvements in efficacy or cell product properties are conceivable, we would certainly look into adopting the technology to move it forward," said Parker.

De Los Angeles also thinks the technology has commercial potential. "I believe that naïve human pluripotent stem cells, either those described in the current study or future versions, will gradually replace the current human pluripotent stem cells," he said. 


\section{Knockout animals}

One key property of naïve iPS cells that is absent from primed human iPS cells is the ability to form germline-competent chimeras. This means that when iPS cells are injected into a blastocyst, the foreign cells are able to differentiate along with the host germ cells and are incorporated into all tissues through development. This property indicates that the cells have full pluripotent potential and allows whole-animal genetic manipulation.

"Besides rodents, no other species has embryonic stem cells that

\section{"We need to look for replication and reproduction of the approach to see if other groups can get the same cell types. Replication is so often not possible." \\ - Chris Parker, Cellular Dynamics International Inc.}

models [as] very valuable for research." contribute to chimerism," said Ding. "In mice and rats, embryonic stem cells can be manipulated, then put back into blastocysts for whole-animal and germline manipulation. If this process of maintaining naïve iPS cells is reproducible and can be applied to other species such as pigs, cows and nonhuman primates, one could imagine genome manipulation of these types of animals to develop disease

Nuwaysir noted that "the cells developed by this process were actually able to form human-mouse chimeras. This is a fundamentally different property that did not exist in iPS cells in the past and raises a lot of new questions about how the cells may be used."

Ding added, "These chimeras may have important implications for the development of humanized animals. The field of humanizing animals is growing because it is very helpful in studying human disease processes, but at this time it is mainly limited to reconstituting specific organs or tissues with human cells."

"This team suggests that it may be possible to incorporate human cells into animals on the whole-system level using the ground-state iPS cells, which could lead to improved mimics of the human system," he said.

One problem, said Ding, is the difficulty in confirming that these human cells are in fact naïve and have chimeric potential.

"Proving that the cells are in this state would require putting them into human blastocysts to see if they incorporate into the tissues, but this will never be possible for humans from an ethical perspective," he said. "The closest way to confirm that this really is a naïve state is to apply the culture conditions to nonhuman primate cells and to see if the naïve monkey cells can contribute to chimerism and integrate into the germline in those animals."

Hanna said that the Weizmann Institute has filed a patent application, and the IP is available for licensing. He expects there could be avenues for commercialization of either the cells themselves or the growth conditions.

Martz, L. SciBX 6(45); doi:10.1038/scibx.2013.1282

Published online Nov. 21, 2013

\section{REFERENCES}

1. Gafni, O. et al. Nature; published online Oct. 30, 2013;

doi:10.1038/nature12745

Contact: Jacob H. Hanna, Weizmann Institute of Science, Rehovot, Israel

e-mail: jacob.hanna@weizmann.ac.il

Contact: Noa Novershtern, same affiliation as above

e-mail: noa.novershtern@weizmann.ac.il

Contact: Rada Massarwa, same affiliation as above e-mail: rada.massarwa@weizmann.ac.il

2. Narsinh, K.H. et al. J. Clin. Invest. 121, 1217-1221 (2011)

3. Hanna, J. et al. Proc. Natl. Acad. Sci. USA 107, 9222-9227 (2010)

4. Tesar, P.J. et al. Nature 448, 196-199 (2007)

COMPANIES AND INSTITUTIONS MENTIONED

Advanced Cell Technology Inc. (OTCBB:ACTC), Santa Monica, Calif. Cellular Dynamics International Inc. (NASDAQ: ICEL), Madison, Wis. Gladstone Institute of Cardiovascular Disease, San Francisco, Calif.

Harvard Medical School, Boston, Mass.

Tel Aviv Sourasky Medical Center, Tel Aviv, Israel

Tel Aviv University Sackler Faculty of Medicine, Tel Aviv, Israel University of California, San Francisco, Calif.

Weizmann Institute of Science, Rehovot, Israel 